\title{
Mechanism of chromatin remodeling and recovery during passage of RNA polymerase II
}

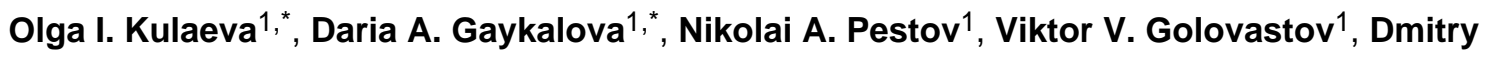 \\ G. Vassylyev ${ }^{2}$, Irina Artsimovitch ${ }^{3}$, and Vasily M. Studitsky ${ }^{1,4}$ \\ 1 Department of Pharmacology, UMDNJ-Robert Wood Johnson Medical School, Piscataway, NJ \\ 08854, USA \\ 2 Department of Biochemistry and Molecular Genetics, University of Alabama at Birmingham, \\ Schools of Medicine and Dentistry, Birmingham, AL 35294, USA \\ ${ }^{3}$ Department of Microbiology, The Ohio State University, Columbus, $\mathrm{OH} 43210$, USA
}

\begin{abstract}
Transcription of eukaryotic genes by RNA polymerase II (Pol II) is typically accompanied by nucleosome survival and minimal exchange of histones $\mathrm{H} 3 / \mathrm{H} 4$. The mechanism of survival and recovery of chromatin structure remains obscure. Here we show how transcription through chromatin by Pol II is uniquely coupled with nucleosome survival. Structural modeling and functional analysis of the intermediates of transcription through a nucleosome was conducted. When Pol II approaches the area of strong DNA-histone interactions, a small intranucleosomal DNA loop (zero-size or Ø-loop) containing transcribing enzyme is formed. During formation of the Ø-loop, the recovery of DNA-histone interactions behind Pol II is tightly coupled with their disruption ahead of the enzyme. This coupling is a distinct feature of the Pol II-type mechanism that allows further transcription through the nucleosome, prevents nucleosome translocation and minimizes displacement of $\mathrm{H} 3 / \mathrm{H} 4$ histones from DNA during enzyme passage.
\end{abstract}

\section{Keywords}

chromatin; nucleosome; RNA polymerase II; transcription; intermediates; structure; mechanism; elongation

Chromatin structure tightly compacts DNA, yet allows efficient progression of DNA and RNA polymerases along the template. Efficient maintenance of nucleosomal organization

\footnotetext{
Users may view, print, copy, and download text and data-mine the content in such documents, for the purposes of academic research, subject always to the full Conditions of use:http://www.nature.com/authors/editorial_policies/license.html\#terms

${ }^{4}$ To whom correspondence should be addressed. studitvm@umdnj.edu; Tel.: 732-235-5240; FAX: 732-235-4073. *Equal contribution

AUTHOR CONTRIBUTIONS

O.I.K. designed and constructed some templates, designed and performed transcription experiments using Pol II; D.A.G. performed characterization and footprinting of the ECs; N.A.P. performed restriction enzyme mapping of the ECs; V.V.G. designed and constructed some templates and performed initial walking experiments; D.G.V. conducted docking; I.A. purified GreB and contributed to writing the manuscript; V.M.S. purified histones and donor chromatin, designed the study, interpreted results and wrote the manuscript.
} 
during passage of RNA Polymerase II (Pol II) is essential for proper gene regulation and cell survival ${ }^{1}$. Recovery of chromatin structure occurs through two different mechanisms (see refs. ${ }^{2},{ }^{3}$ for review). During intense transcription, partial loss ${ }^{4}{ }^{8}$ and exchange ${ }^{9}{ }^{14}$ of all core histones at the transcribed regions were reported. In contrast, on moderately transcribed genes, fast and extensive transcription-dependent exchange of $\mathrm{H} 2 \mathrm{~A} / \mathrm{H} 2 \mathrm{~B}$, but not $\mathrm{H} 3 / \mathrm{H} 4$, histones was observed ${ }^{9}{ }^{14}$.

The Pol II-type mechanism of transcription through chromatin in vitro is conserved from yeast to human ${ }^{15}$. It is characterized by a high nucleosomal barrier to transcription ${ }^{15}{ }^{17}$, and by displacement of a single H2A/H2B dimer ${ }^{17}{ }^{19}$ that matches the apparent effect of Pol II passage in vivo ${ }^{11},{ }^{20}$. The subnucleosome (DNA-bound histone hexamer formed upon release of $\mathrm{H} 2 \mathrm{~A} / \mathrm{H} 2 \mathrm{~B}$ dimer from the octamer) survives Pol II passage through a nucleosome and remains at the original position on DNA ${ }^{17}$. A considerably different, Pol III-type mechanism, involves transfer of a complete histone octamer from in front of the transcribing enzyme to behind $\mathrm{it}^{21}{ }^{23}$.

Nucleosomes positioned on DNA sequences having a high affinity for histones (HA sequences) present a polar barrier to transcription by Pol II in vitro ${ }^{15}$. In one (nonpermissive) orientation, the nucleosomal barrier is high, whereas in the opposite (permissive) orientation the barrier is much lower. In the present study, we have studied sequence determinants of the barrier and structures of the intermediates formed during Pol II transcription through chromatin. This analysis supports a model in which a small intranucleosomal DNA loop (Ø-loop) containing transcribing Pol II is formed during transcription through a nucleosome. Formation of the $\varnothing$-loop enables nucleosomes to survive transcription without displacement of core histones from DNA and could play a key role in preservation of the histone code during Pol II transcription.

\section{RESULTS}

\section{HA sequences dictate polarity of the nucleosomal barrier to Pol II}

First, we evaluated the possibility that the polar barrier to Pol II transcription ${ }^{15},{ }^{24}$ is dictated by asymmetric location of the high affinity (HA) sequences within the nucleosome. We aligned 601, 603 and 605 nucleosome positioning sequences ${ }^{25}$ and identified the HA sequences ${ }^{26}$. In all cases, the HA sequences are localized highly asymmetrically and are more similar to the consensus within the promoter-distal half of nucleosomal DNA in the non-permissive orientations than in the permissive orientations (Supplementary Fig. 1).

To dissect the effects of different sequence elements on their affinity for core histones and on nucleosome positioning, we mutated various regions of the 603R template (Supplementary Fig. 1) and analyzed the resulting nucleosomes by native PAGE (Fig. 1a). Mutations in the consensus-like sequences in the promoter-proximal, left (-L) half of the template 603R-L barely affected the affinity or the positioning properties of the 603R template. Similar changes introduced in the right half (603R-R template) decreased the DNA affinity for core histones and resulted in the loss of nucleosome positioning (Fig. 1a). Thus HA sequences located in the distal half of the 603R template dictate both DNA-histone affinity and nucleosome positioning. Partial mutagenesis of the right half (603R-R(2-3) 
template) also resulted in decreased affinity of the template for core histones, but did not interfere with nucleosome positioning. Thus the determinants for nucleosome positioning and DNA-histone affinity are distinct.

Next, we evaluated the effect of the HA sequences on transcription by Pol II. We conducted these experiments using mononucleosomal 603R templates ${ }^{15},{ }^{17}$ presenting a high, polar barrier to Pol II progression ${ }^{15}$. Positioned 603R nucleosomes were ligated downstream of pre-assembled elongation complexes EC-119 (the numerical indices indicate the position of the Pol II active center on the template relative to promoter-proximal nucleosomal DNA boundary). Then nascent RNA was pulse-labeled by forming EC-83 and transcription was resumed in the presence of unlabeled NTPs ${ }^{17}$ (Supplementary Fig. 2a). We used the fraction of Pol II molecules that reach the end of the template (run-off) to quantify the height of the barrier, which is located primarily in the +45 region of nucleosomal DNA. We used a similar approach to study transcription through chromatin by E. coli RNAP (Supplementary Fig. 2b).

Mutations introduced into the critical HA sequences (603R-R template) resulted in a much higher fraction of templates transcribed to completion, as compared with the 603R template (65\% and $32 \%$ at $300 \mathrm{mM} \mathrm{KCl}$, respectively; Fig. $1 \mathrm{~b}, \mathrm{c}$ ). Thus, the $-\mathrm{R}$ mutations convert the non-permissive $603 \mathrm{R}$ template into the permissive $603 \mathrm{R}-\mathrm{R}$ template. In contrast, the transcriptional properties of the $603 \mathrm{R}$ and 603R-L templates are nearly identical. The mutations in the $-\mathrm{R}(2-3)$ sequences (Fig. 1c) result in strong relief of the barrier without affecting nucleosome positioning (Fig. 1a). Thus, the high affinity of the $-\mathrm{R}(2-3)$ sequences for histones dictates a strong nucleosomal barrier to transcription.

In summary, these experiments suggest that, surprisingly, the critical DNA sequences that confer the high nucleosomal barrier to Pol II transcription (the HA sequences) are located more than $40 \mathrm{bp}$ downstream of the active center of the enzyme arrested at the +45 region (Supplementary Fig. 1).

\section{Modeling Pol II elongation complexes in a nucleosome: a Ø-loop}

How can DNA sequences located far downstream of Pol II induce its arrest in the +45 region of the nucleosome? Our previous studies suggested that during productive transcription, Pol II localized at the +45 region induces uncoiling of nucleosomal DNA from the octamer to allow further transcription ${ }^{15}$. We propose that as the Pol II molecule transcribes through the +45 region, it can form a tight intranucleosomal DNA loop containing the active enzyme (Fig. 1d (1)). This loop was named a "zero-size" loop (Øloop) because it is so small that the original, "pre-transcriptional" DNA-histone interactions are formed both in front of and behind transcribing Pol II. Formation of the Ø-loop would result in steric interference between Pol II molecule and the promoter-distal end of the nucleosomal DNA. This, in turn, could induce partial uncoiling of DNA from the octamer ahead of Pol II and facilitate further progression through a permissive nucleosome (Fig. 1d, (2) and (3)). Conversely, downstream HA sequences could prevent DNA uncoiling and thus hinder further transcription through a non-permissive nucleosome (1). Formation of a similar $\varnothing$-loop was observed in our studies of bacteriophage SP6 RNA polymerase (RNAP) stalled at the +45 region $^{27}$. 
To evaluate the possibility of $\emptyset$-loop formation by Pol II, we modeled the $\varnothing$-loop by docking the high-resolution structures of yeast Pol II EC onto the nucleosome (PDB IDs 1 aoi and $1 \mathrm{y} 1 \mathrm{w}$, see refs. ${ }^{28},{ }^{29}$ ) (Fig. 2). This analysis suggests that the $\varnothing$-loop can be formed only when Pol II is at the position +39 or +49 in a nucleosome and at least 50 bp are displaced from the promoter-distal end of nucleosomal DNA.

This is since the $\varnothing$-loop-containing EC+39 has the following properties (Fig. 2). (i) The bulk of the Pol II molecule faces into solution and there are no steric clashes with core histones.

(ii) The $90^{\circ}$ DNA bend present in the EC faces the octamer surface and allows formation of the Ø-loop. (iii) DNA-histone contacts with 20-bp DNA region behind the EC stabilize the $\emptyset$-loop. (iv) Displacement of $250 \mathrm{bp}$ from the promoter-distal end of the nucleosome reduces the size of the DNA region interacting with histones in front of the enzyme from $\sim 100$ to $50 \mathrm{bp}$. This would facilitate further uncoiling of DNA from the octamer ahead of Pol II and transcription through the nucleosome. (v) The R3 HA DNA sequence (Supplementary Fig. 1) is localized within the displaced 50-bp DNA region, and would be expected to interfere with DNA displacement and to trigger Pol II arrest in the +45 region. (vi) The modeling identified a negatively charged region on the surface of Pol II that could be important for proper transcription through chromatin (Supplementary Fig. 3 and Supplementary Discussion).

Formation of the $\varnothing$-loop is possible only in one rotational orientation of the EC on DNA (at positions +39 or +49 ). Movement of the enzyme by $1 \mathrm{nt}$ would result in a $\sim 36^{\circ}$ rotation around the DNA axis and steric clashes between Pol II and the histone octamer. Thus, Pol II translocation after formation of the Ø-loop would disrupt the DNA-histone interactions upstream and/or downstream of the enzyme. If only the downstream histone-DNA interactions are broken (Fig. 1d (3)), Pol II could transcribe through chromatin without displacement of the octamer into solution, as was previously observed experimentally ${ }^{17}$.

\section{Structures of Pol II-nucleosome complexes: formation of the Ø-loop}

We evaluated the proposed model (Fig. 1d) by footprinting of ECs stalled at various positions within permissive 603 nucleosomes. It is extremely technically challenging to obtain large quantities of homogeneous Pol II ECs stalled at a desired position in a nucleosome. All general aspects of the Pol II-type mechanism are recapitulated by E. coli $\operatorname{RNAP}^{30}$ but not by other previously analyzed RNAPs ${ }^{15},{ }^{21},{ }^{23}$. Since homogeneous $E$. coli ECs can be obtained in sufficient amounts, we used them for the initial analysis of the Pol II-type mechanism of transcription through chromatin.

Our modeling suggests that the Ø-loop could be formed when RNAP transcribes 39 or 49 bp of nucleosomal DNA. Therefore, we analyzed a subset of ECs halted near these positions on the 603 template (positions $-39,-5,+42$ or +49 , Supplementary Fig. 4 a). To map the position of the active center of RNAP in the ECs, they were incubated in the presence of GreB. GreB strongly facilitates RNA cleavage by E. coli RNAP; the cleavage reaction is mediated by the RNAP active site and occurs only in complexes formed after backtracking of the enzyme ${ }^{31}{ }^{33}$. We found that EC+49 complexes were resistant to GreB (Supplementary Fig. 4b). When stalled at +42 , RNAP backtracked by $1-2$ nt to form EC +41 . 
Therefore in both complexes the active center remains in close proximity to the $3^{\prime}$-end of the RNA and the RNAP is not arrested.

The structures of these complexes were analyzed using endonuclease DNaseI (Fig. 3a). As expected, each stalled EC (e.g. EC-39) protected 30-bp, and the nucleosome protected $\sim 150$-bp from DNaseI digestion (Fig. 3b). When RNAP forms EC+41, the nucleosomal DNA is completely uncoiled from the octamer upstream of the RNAP (Fig. 3b,c). The DNA downstream of the EC remains fully bound but is distorted around the +90 and +100 positions, as shown by the appearance of hypersensitive sites.

Although the EC+49 is stalled inside the nucleosome, the nucleosome-specific features of the footprint persist on the majority ( $270 \%$ ) of the complexes. In EC+49, DNA protection by the EC was not easily discernible, likely because the nucleosome-specific DNA protection masks protection by the EC. However, we argue that the EC+49 should remain active during the 30-sec digestion with DNaseI, because most complexes produced run-off transcripts (Supplementary Fig. 4 and Supplementary Discussion). Thus the persistence of the nucleosome-specific DNA protection pattern in EC+49 suggests that the original DNAhistone contacts are re-formed both upstream and downstream of the stalled RNAP. This is possible only if the EC+49 forms the Ø-loop on the surface of the histone octamer (the closed intermediate, Fig. 3d). Therefore our data support the idea that RNAP forms the $\varnothing$ loop at the position +49 .

Although the nucleosome-specific features predominate in EC+49, DNA both upstream and downstream of the EC is more accessible to DNaseI than in the original nucleosome. Thus quantitative analysis (Fig. 3d) revealed that the accessibility of nucleosomal DNA upstream of RNAP (+20 to +30 region) is less pronounced than it is downstream of the enzyme. A short DNA region at the promoter-proximal end of the nucleosomal DNA $(+1$ to +20$)$ is almost completely resistant to DNase I. Finally, multiple DNA sites downstream of EC+49 are accessible to DNaseI to a similar degree, but considerably less than in histone-free DNA. Most likely, both upstream and downstream contacts are lost in the same ternary complex (Fig. 3d, intermediate 2); it is otherwise difficult to explain how the contacts between +20 and +30 can be disrupted without displacing the +1 to +20 region at the end of nucleosomal DNA. Together, these data imply that DNA is uncoiled from the octamer in front of the enzyme on $\mathbf{3 0 \%}$ of templates (forming "open" intermediate 1) and on an even a smaller ( $\triangle 0 \%$ ) fraction of templates, the nucleosomal DNA is partially uncoiled from the octamer behind RNAP (2). The +1 to +20 region remains associated with the octamer. Since the histone octamer is not lost, the intermediates in Figure $3 \mathrm{~d}$ are most likely in rapid equilibrium.

The data suggest a pathway for transcription through a nucleosome (Fig. 3e). As RNAP enters the nucleosome, it initially uncoils nucleosomal DNA primarily behind itself, as seen in EC+41. As the enzyme reaches the +49 position, the DNA behind RNAP is re-coiled on the surface of the octamer, the Ø-loop is formed, and the DNA in front of the complex becomes partially uncoiled from the octamer (EC+49). Sequential release of DNA-histone contacts in the $\varnothing$-loop intermediate allows both unimpeded transcription (through selective 
disruption of the downstream interactions) and nucleosome recovery (through re-formation of the original DNA-histone interactions upstream of RNAP).

To confirm that E. coli RNAP and yeast Pol II form similar complexes during transcription through a nucleosome, Pol II was stalled at positions $-5,+41$ or +49 in the 603 nucleosome and DNA accessibility within the complexes were analyzed using restriction enzymes (Fig. 4a). Nucleosomes strongly protect DNA from digestion with restriction enzymes ${ }^{34}$; thus both the Cac8I and StyI intranucleosomal sites are protected from digestion in EC-5 (Fig. 4b) and in intact nucleosomes (not shown). As expected, in EC+41, the DNA behind Pol II (the $\mathrm{Cac} 8 \mathrm{I}$ site) is sensitive to digestion and the DNA in front of the enzyme (the StyI site) is resistant (Fig. 4b and Supplementary Fig. 5). Importantly, the StyI site is located far downstream of the Pol II boundary on the DNA ${ }^{35}$. In contrast, in EC+49, the Cac8I site is largely protected and the StyI site is accessible. These data suggest that yeast Pol II and $E$. coli RNAP induce similar structural rearrangements of DNA/histone contacts during transcription through nucleosomes.

In summary, our data suggest that the structures of the intermediates formed before and after Pol II reaches the position +49 are very different: initially nucleosomal DNA is displaced upstream of Pol II, but distal to position +49 , DNA displacement occurs primarily downstream of the enzyme (Fig. 3e). Thus, formation of the Ø-loop at the +49 position constitutes the transition point that allows nucleosome recovery at the original position on the DNA.

\section{Opposite effects of the two H2A/H2B dimers on the barrier to Pol II}

Our next goal was to evaluate functional predictions of our working model (Fig. 3e). The asymmetric roles of the histone-DNA contacts are inherent in the model. Removal of the distal histone H2A/H2B dimer (D-dimer) would result in release of the promoter-distal end of nucleosomal DNA into solution, facilitating formation of the Ø-loop (Fig. 5a (1) and (2) ) and transcription through the nucleosome (3). In contrast, removal of the proximal P-dimer would eliminate DNA-binding site upstream of the EC and therefore would strongly destabilize the $\emptyset$-loop $\left(2^{\prime}\right)$. In the latter case, two scenarios are possible: (a) If the upstream contacts are not essential for further transcription, Pol II would displace downstream DNA and continue. (b) Alternatively, if the upstream contacts are essential, removal of the Pdimer would cause arrest at the +45 region. Thus the model predicts that removal of the promoter-proximal or the promoter-distal dimer could have drastically different impacts on the +45 nucleosomal barrier (Fig. 5a).

To evaluate these possibilities, permissive 603 nucleosomes and subnucleosomes missing either the P- or the D-dimer (-P- and -D-hexasomes, respectively) were constructed (Supplementary Fig. 6) and transcribed by Pol II (Fig. 5b). As expected, removal of the promoter-distal D-dimer resulted in a partial relief of the +45 barrier (Fig. $5 \mathrm{c}$ ).

In contrast, removal of the promoter-proximal P-dimer results in a strong increase (8- and 12 -fold at 40 and $150 \mathrm{mM} \mathrm{KCl}$, respectively) in the strength of the +45 barrier (Fig. $5 \mathrm{c}$ ). Thus, removal of the P-dimer transforms the permissive nucleosome into a non-permissive 
one. Therefore the DNA-histone contacts upstream of the EC paused at the +45 region are essential for further transcription through the nucleosome.

In summary, the P- and D-dimers play different roles within the same nucleosome. As expected, removal of the P-dimer results in a strong Pol II arrest in the +45 region, most likely because the Ø-loop cannot form, the promoter-distal end of nucleosomal DNA cannot be displaced, and transcription is hindered (Fig. 5a). In contrast, removal of the D-dimer results in a modest relief of the +45 barrier, most likely because displacement of the promoter-distal end of the nucleosomal DNA and formation of the Ø-loop are facilitated.

\section{DISCUSSION}

Our structural analysis of the ECs formed during transcription through a nucleosome (Figs. 2-4) suggests a new mechanism of transcription through chromatin (Fig. 3e). This mechanism is consistent with the strong effects of far downstream sequences on Pol II pausing in the +45 intranucleosomal DNA region (Fig. 1) and the requirement of the promoter-proximal histone $\mathrm{H} 2 \mathrm{~A} / \mathrm{H} 2 \mathrm{~B}$ dimer for efficient Pol II transcription through the nucleosome (Fig. 5).

This mechanism operates on various DNA sequences (Supplementary Discussion) and relies on a feedback loop to couple nucleosome survival to efficient transcription through chromatin via conformational changes in the nucleosome structure (Fig. 6a): if a nucleosome cannot survive transcription, Pol II becomes arrested. Since the key features of the process of transcription through a nucleosome by yeast and human Pol II are highly similar ${ }^{15}$, the mechanism may well be conserved in all eukaryotes.

The differences in the structures of the intermediates formed by the Pol II- and Pol III-type mechanisms could explain the different nucleosome fates during these processes

(Supplementary Figs. 7-8 and Supplementary Discussion). At the same time, the Ø-loop can be formed during transcription through the +45 region by both mechanisms ${ }^{27}$. These observations suggest that the conformational dynamics involved in formation of the Ø-loop are intrinsic to nucleosomes. Therefore the conformational changes in nucleosomal structure observed during transcription are likely to occur during progression of other processive enzymes (e.g. ATP-dependent remodelers and DNA polymerases) through chromatin.

Many eukaryotic genes are regulated at the level of transcript elongation and nucleosomes are likely to be key players in this regulation ${ }^{36}{ }^{39}$. Our observations suggest that inefficient resolution of the Ø-loop can cause strong nucleosome-specific pausing and the paused intermediate could be a target for regulation of the rate of elongation through chromatin. Such regulation could be mediated by variable promoter-distal sequence of nucleosomal DNA and/or by histone chaperones (e.g. FACT ${ }^{17},{ }^{18},{ }^{40}{ }^{42}$ ) facilitating displacement of the distal D-dimer.

In contrast, the proximal P-dimer is likely to be essential for both nucleosome survival and efficient transcription. Displacement of the P-dimer leads to arrest of Pol II within the nucleosome (Fig. 5) and most likely occurs only transiently in vivo. Since both H2A/H2B dimers are exchanged during Pol II transcription in vivo ${ }^{11}$, the P-dimer must be immediately 
replaced to avoid arrest of transcribing Pol II and/or loss of the histone octamer. Therefore, our data argue that a transcription-coupled process could guarantee fast rebinding of the displaced $\mathrm{H} 2 \mathrm{~A} / \mathrm{H} 2 \mathrm{~B}$ dimer(s) to nucleosomes. Factors facilitating P-dimer re-binding are expected to facilitate transcription through chromatin. Indeed, the $\mathrm{H} 2 \mathrm{~A} / \mathrm{H} 2 \mathrm{~B}$ chaperone FACT associates with elongating Pol II, contributes to nucleosome survival during transcription in vivo ${ }^{18},{ }^{40},{ }^{41}$, and facilitates transcription through nucleosomes in vitro ${ }^{15},{ }^{18}$.

Recent studies suggest that on moderately Pol II-transcribed genes exchange of $\mathrm{H} 3 / \mathrm{H} 4$ histones is at least 20-fold slower than that of $\mathrm{H} 2 \mathrm{~A} / \mathrm{H} 2 \mathrm{~B}$ (see Introduction). Our work suggests that $\mathrm{H} 3 / \mathrm{H} 4$ are not exchanged because they are never completely displaced from the DNA during $\varnothing$-loop-mediated transcription through chromatin. Since most eukaryotic genes are transcribed at moderate levels, transcription-dependent exchange of bulk H3/H4 histones is considerably slower than $\mathrm{H} 2 \mathrm{~A} / \mathrm{H} 2 \mathrm{~B}$ dimer exchange ${ }^{20}$.

Pol II transcription through chromatin is coupled with nucleosome survival at the original position on DNA. Why is nucleosome survival at the original position important? Transcription of a eukaryotic gene using the alternative, Pol III-type mechanism would trigger an extensive displacement/exchange of all core histones ${ }^{21}$. On the contrary, Pol IItype mechanism involves only minimal exchange of histones H3/H4 (Fig. 6b). Since the H3/H4 histones contain the majority of the sites for post-translational modifications, including some epigenetic marks ${ }^{43}$, the Pol II-type mechanism could specifically allow for the survival of the original $\mathrm{H} 3 / \mathrm{H} 4$ histones and their covalent modifications during transcription. Since almost the entire eukaryotic genome is transcribed at a certain non-zero frequency ${ }^{44},{ }^{45}$, this mechanism could mediate maintenance of epigenetic marks across the genome.

In summary, transcription of the majority of eukaryotic genes by Pol II is accompanied by minimal disruption of chromatin structure likely ensuring maintenance of genome stability ${ }^{1}$. In particular, the minimal exchange of $\mathrm{H} 3 / \mathrm{H} 4$ histones may well guarantee the survival of epigenetic "marks" and other post-translational modifications of H3/H4 histones. Our analysis suggests that the Ø-loop intermediate mediates both the efficient Pol II transcription through chromatin and the preservation of the $\mathrm{H} 3 / \mathrm{H} 4$ code. Formation of $\varnothing$-loops is tightly controlled through a feedback mechanism and may turn out to be extensively regulated.

\section{METHODS}

\section{Protein purification}

We purified hexahistidine-tagged E. coli RNAP, Pol II, core histones and GreB protein using published protocols ${ }^{17},{ }^{46}, 47$.

\section{DNA templates and sequence alignment}

The 603 and 603R templates for Pol II were described previously ${ }^{15}$. We prepared the variants of the 603R template (603R, 603R-L and -R) by annealing pairs of long overlapping oligonucleotides and filling-in with the Klenow fragment of DNA polymerase I (NEB). The double-stranded DNA fragments were then PCR-amplified using a different pair of primers to obtain 201-bp fragments. The 201-bp fragments were gel-purified and PCR-amplified 
with another pair of primers to obtain 262-bp fragments containing $T s p$ R1 site. After digestion with $T s p$ RI (NEB) 249-bp fragments were obtained. The sequences of the variant 603R templates will be provided on request. We obtained the 110-bp DNA fragments for reconstitution of hexasomes and tetrasome PCR-amplification of the plasmid pGEM-3Z/ $603^{26}$ with different pairs of primers, followed by $T s p \mathrm{R} 1$ digestion.

To obtain the 603-42 and 603-49 templates for Pol II, we mutated the original 603 template at four or six positions to allow stalling of Pol II at +42 or +49 positions in the 603 nucleosome, respectively. The nucleosome positioning sequences were amplified by PCR and digested with $T s p$ RI (NEB) to obtain the 149-bp DNA fragment.

\section{Reconstitution of nucleosomes and subnucleosomes}

We gel-purified the 149-bp and 249-bp DNA fragments, used them for nucleosome reconstitution by octamer exchange at 1:3 DNA:chromatin ratio ${ }^{17}$. The hexasomes were reconstituted using chicken erythrocytes core histones by dialysis from $2 \mathrm{M} \mathrm{NaCl}^{48}$.

To obtain 603-42 and 603-49 templates for E. coli RNAP we mutated the original 603 template to replace four or six nucleotides in DNA and allow stalling of RNAP at the +42 or +49 positions within the 603 nucleosome, respectively. We amplified the nucleosome positioning sequences by PCR, digested by $T s p$ RI (NEB) and ligated through the $T s p$ RI site to a T7A1 promoter-bearing fragment ${ }^{30}$. Ligated products were re-amplified with one $5^{\prime}$ end-labeled primer, gel-purified, and assembled into nucleosomes. We reconstituted nucleosomes on the DNA templates by histone octamer transfer from chicken -H1 erythrocyte donor chromatin ${ }^{17}$.

\section{Transcription of nucleosomes and subnucleosomes}

E. coli RNAP: We formed elongation complexes containing 11-mer RNA (EC-39) on preassembled nucleosomal templates as described ${ }^{30}$. In experiments with labeled RNA EC-39 was pulse-labeled in the presence of [a- $\left.{ }^{32} \mathrm{P}\right]-\mathrm{GTP}(3000 \mathrm{Ci} / \mathrm{mmol}$, PerkinElmer Life Sciences). EC-39 was then extended in the presence of a subset of NTPs to form EC-5. In footprinting experiments, all steps were performed in solution. In experiments involving labeled RNA and GreB treatment, EC-5 was immobilized on Ni-NTA-agarose ${ }^{30}$. After extensive washes the complexes were eluted from Ni-NTA beads in the presence of $100 \mathrm{mM}$ imidazole and transcription was continued in solution. EC-5, EC+41 or EC+49 were formed in the presence of $1 \mu \mathrm{M}$ ATP on the 603-42 or 603-49 templates. EC-5 was further extended in the presence of $300 \mu \mathrm{M} \mathrm{CTP}$, UTP, GTP and $150 \mu \mathrm{M} 3^{\prime} 25^{\circ} \mathrm{C}$ for $4 \mathrm{~min}$ or in the presence of $200 \mu \mathrm{M}$ of all NTPs in TB300. Labeled RNA was purified and separated by denaturing PAGE. Transcription by Pol II was performed as described ${ }^{17}, 30$.

\section{DNasel footprinting}

We carried out DNase I footprinting at a final concentration of end-labeled templates of 2.5 $\mu \mathrm{g} / \mathrm{ml}$ in the presence of 10 -fold weight excess of unlabeled $-\mathrm{H} 1$ chicken erythrocyte chromatin in TB100 (20 mM Tris $\mathrm{HCl} \mathrm{pH} 8.0,5 \mathrm{mM} \mathrm{MgCl}_{2}, 2 \mathrm{mM} \beta$-ME, $100 \mathrm{mM} \mathrm{KCl}$ ). DNaseI was added to a final concentration $20-50 \mathrm{U} / \mathrm{ml}$ for $30 \mathrm{sec}$ at $37^{\circ} \mathrm{C}$ after formation of the desired ECs. The reactions were terminated by adding EDTA to $10 \mathrm{mM}$. The samples 
were resolved in a native ge ${ }^{17}$. Gel fragments containing desired complexes were cut, DNA extracted, purified and analyzed by denaturing PAGE. The gels were quantified using a PhosphorImager.

\section{Analysis of Pol II elongation complexes using the restriction enzyme sensitivity assay}

To stall Pol II at +42 and +49 positions in the 603 nucleosome, we assembled EC-39, immobilized it on Ni-NTA-agarose ${ }^{17}$. Then we ligated EC-39 or 2 hours at $16^{\circ} \mathrm{C}$ in TB40 to nucleosomes assembled on the 149-bp DNA fragment, washed, eluted into solution ${ }^{17}$ and extended in the presence of a subset of NTPs to form EC-5, EC+41 or EC+49 in TB300 at $20^{\circ} 6 \mathrm{C}$ (Supplementary Methods). The complexes were diluted 3-fold with TB0 to decrease concentration of $\mathrm{KCl}$ to $100 \mathrm{mM}$, digested with the restriction enzymes indicated, and separated by native PAGE. The gels were quantified using a PhosphorImager.

\section{Supplementary Material}

Refer to Web version on PubMed Central for supplementary material.

\section{Acknowledgments}

We thank John Widom (Northwestern University) for plasmids containing the nucleosome positioning sequences, Guohui Zheng for making the movie, and David Clark, Mark Gartenberg, Jeffrey Hayes and Don Luse for suggestions and critical reading of the manuscript. This work was supported by NSF 0549593 and NIH GM58650 grants to V.M.S., NIH GM74252 and GM74840 grants to D.G.V., and NIH GM067153 grant to I.A.

\section{References}

1. Martens JA, Wu PY, Winston F. Regulation of an intergenic transcript controls adjacent gene transcription in Saccharomyces cerevisiae. Genes Dev. 2005; 19:2695-2704. [PubMed: 16291644]

2. Thiriet $\mathrm{C}$, Hayes JJ. Histone dynamics during transcription: exchange of $\mathrm{H} 2 \mathrm{~A} / \mathrm{H} 2 \mathrm{~B}$ dimers and H3/H4 tetramers during pol II elongation. Results Probl Cell Differ. 2006; 41:77-90. [PubMed: 16909891]

3. Kulaeva OI, Gaykalova DA, Studitsky VM. Transcription through chromatin by RNA polymerase II: Histone displacement and exchange. Mutat Res. 2007; 618:116-129. [PubMed: 17313961]

4. Kristjuhan A, Svejstrup JQ. Evidence for distinct mechanisms facilitating transcript elongation through chromatin in vivo. EMBO J. 2004; 23:4243-4252. [PubMed: 15457216]

5. Lee CK, Shibata Y, Rao B, Strahl BD, Lieb JD. Evidence for nucleosome depletion at active regulatory regions genome-wide. Nat Genet. 2004; 36:900-905. [PubMed: 15247917]

6. Schwabish MA, Struhl K. Evidence for eviction and rapid deposition of histones upon transcriptional elongation by RNA polymerase II. Mol Cell Biol. 2004; 24:10111-10117. [PubMed: 15542822]

7. Petesch SJ, Lis JT. Rapid, transcription-independent loss of nucleosomes over a large chromatin domain at Hsp70 loci. Cell. 2008; 134:74-84. [PubMed: 18614012]

8. Zhao J, Herrera-Diaz J, Gross DS. Domain-wide displacement of histones by activated heat shock factor occurs independently of Swi/Snf and is not correlated with RNA polymerase II density. Mol Cell Biol. 2005; 25:8985-8999. [PubMed: 16199876]

9. Wirbelauer C, Bell O, Schubeler D. Variant histone H3.3 is deposited at sites of nucleosomal displacement throughout transcribed genes while active histone modifications show a promoterproximal bias. Genes Dev. 2005; 19:1761-1766. [PubMed: 16077006]

10. Schwartz BE, Ahmad K. Transcriptional activation triggers deposition and removal of the histone variant H3.3. Genes Dev. 2005; 19:804-814. [PubMed: 15774717] 
11. Thiriet C, Hayes JJ. Replication-independent core histone dynamics at transcriptionally active loci in vivo. Genes Dev. 2005; 19:677-682. [PubMed: 15769942]

12. Dion MF, et al. Dynamics of replication-independent histone turnover in budding yeast. Science. 2007; 315:1405-1408. [PubMed: 17347438]

13. Rufiange A, Jacques PE, Bhat W, Robert F, Nourani A. Genome-wide replication-independent histone $\mathrm{H} 3$ exchange occurs predominantly at promoters and implicates $\mathrm{H} 3 \mathrm{~K} 56$ acetylation and Asf1. Mol Cell. 2007; 27:393-405. [PubMed: 17679090]

14. Jamai A, Imoberdorf RM, Strubin M. Continuous histone H2B and transcription-dependent histone H3 exchange in yeast cells outside of replication. Mol Cell. 2007; 25:345-355. [PubMed: 17289583]

15. Bondarenko VA, et al. Nucleosomes can form a polar barrier to transcript elongation by RNA polymerase II. Mol Cell. 2006; 24:469-479. [PubMed: 17081995]

16. Izban MG, Luse DS. Transcription on nucleosomal templates by RNA polymerase II in vitro: inhibition of elongation with enhancement of sequence-specific pausing. Genes Dev. 1991; 5:683696. [PubMed: 2010092]

17. Kireeva ML, et al. Nucleosome remodeling induced by RNA polymerase II: loss of the H2A/H2B dimer during transcription. Mol Cell. 2002; 9:541-552. [PubMed: 11931762]

18. Belotserkovskaya R, et al. FACT facilitates transcription-dependent nucleosome alteration. Science. 2003; 301:1090-1093. [PubMed: 12934006]

19. Angelov D, et al. Nucleolin is a histone chaperone with FACT-like activity and assists remodeling of nucleosomes. EMBO J. 2006; 25:1669-1679. [PubMed: 16601700]

20. Kimura H, Cook PR. Kinetics of core histones in living human cells: little exchange of $\mathrm{H} 3$ and $\mathrm{H} 4$ and some rapid exchange of H2B. J Cell Biol. 2001; 153:1341-1353. [PubMed: 11425866]

21. Studitsky VM, Clark DJ, Felsenfeld G. A histone octamer can step around a transcribing polymerase without leaving the template. Cell. 1994; 76:371-382. [PubMed: 8293470]

22. Studitsky VM, Clark DJ, Felsenfeld G. Overcoming a nucleosomal barrier to transcription. Cell. 1995; 83:19-27. [PubMed: 7553869]

23. Studitsky VM, Kassavetis GA, Geiduschek EP, Felsenfeld G. Mechanism of transcription through the nucleosome by eukaryotic RNA polymerase. Science. 1997; 278:1960-1963. [PubMed: 9395401]

24. Hall MA, et al. High-resolution dynamic mapping of histone-DNA interactions in a nucleosome. Nat Struct Mol Biol. 2009; 16:124-129. [PubMed: 19136959]

25. Morozov AV, et al. Using DNA mechanics to predict in vitro nucleosome positions and formation energies. Nucleic Acids Res. 2009; 37:4707-4722. [PubMed: 19509309]

26. Thastrom A, Bingham LM, Widom J. Nucleosomal locations of dominant DNA sequence motifs for histone-DNA interactions and nucleosome positioning. J Mol Biol. 2004; 338:695-709. [PubMed: 15099738]

27. Bednar J, Studitsky VM, Grigoryev SA, Felsenfeld G, Woodcock CL. The nature of the nucleosomal barrier to transcription: direct observation of paused intermediates by electron cryomicroscopy. Mol Cell. 1999; 4:377-386. [PubMed: 10518218]

28. Luger K, Mader AW, Richmond RK, Sargent DF, Richmond TJ. Crystal structure of the nucleosome core particle at 2.8 A resolution. Nature. 1997; 389:251-260. [PubMed: 9305837]

29. Kettenberger H, Armache KJ, Cramer P. Complete RNA polymerase II elongation complex structure and its interactions with NTP and TFIIS. Mol Cell. 2004; 16:955-965. [PubMed: 15610738]

30. Walter W, Kireeva ML, Studitsky VM, Kashlev M. Bacterial polymerase and yeast polymerase II use similar mechanisms for transcription through nucleosomes. J Biol Chem. 2003; 278:3614836156. [PubMed: 12851391]

31. Laptenko O, Lee J, Lomakin I, Borukhov S. Transcript cleavage factors GreA and GreB act as transient catalytic components of RNA polymerase. EMBO J. 2003; 22:6322-6334. [PubMed: 14633991]

32. Borukhov S, Sagitov V, Goldfarb A. Transcript cleavage factors from E. coli. Cell. 1993; 72:459466. [PubMed: 8431948] 
33. Komissarova N, Kashlev M. RNA polymerase switches between inactivated and activated states by translocating back and forth along the DNA and the RNA. J Biol Chem. 1997; 272:15329-15338. [PubMed: 9182561]

34. Polach KJ, Widom J. Restriction enzymes as probes of nucleosome stability and dynamics. Methods Enzymol. 1999; 304:278-298. [PubMed: 10372366]

35. Gnatt AL, Cramer P, Fu J, Bushnell DA, Kornberg RD. Structural basis of transcription: an RNA polymerase II elongation complex at 3.3 A resolution. Science. 2001; 292:1876-1882. [PubMed: 11313499]

36. Sullivan EK, Weirich CS, Guyon JR, Sif S, Kingston RE. Transcriptional activation domains of human heat shock factor 1 recruit human SWI/SNF. Mol Cell Biol. 2001; 21:5826-5837. [PubMed: 11486022]

37. Guenther MG, Levine SS, Boyer LA, Jaenisch R, Young RA. A chromatin landmark and transcription initiation at most promoters in human cells. Cell. 2007; 130:77-88. [PubMed: 17632057]

38. Mavrich TN, et al. Nucleosome organization in the Drosophila genome. Nature. 2008; 453:358362. [PubMed: 18408708]

39. Core LJ, Waterfall JJ, Lis JT. Nascent RNA sequencing reveals widespread pausing and divergent initiation at human promoters. Science. 2008; 322:1845-1848. [PubMed: 19056941]

40. Formosa T, et al. Defects in SPT16 or POB3 (yFACT) in Saccharomyces cerevisiae cause dependence on the Hir/Hpc pathway: polymerase passage may degrade chromatin structure. Genetics. 2002; 162:1557-1571. [PubMed: 12524332]

41. Pavri R, et al. Histone H2B monoubiquitination functions cooperatively with FACT to regulate elongation by RNA polymerase II. Cell. 2006; 125:703-717. [PubMed: 16713563]

42. van Holde KE, Lohr DE, Robert C. What happens to nucleosomes during transcription? J Biol Chem. 1992; 267:2837-2840. [PubMed: 1310672]

43. Kouzarides T. Chromatin modifications and their function. Cell. 2007; 128:693-705. [PubMed: 17320507]

44. David L, et al. A high-resolution map of transcription in the yeast genome. Proc Natl Acad Sci USA. 2006; 103:5320-5325. [PubMed: 16569694]

45. Araki R, et al. More than 40,000 transcripts, including novel and noncoding transcripts, in mouse embryonic stem cells. Stem Cells. 2006; 24:2522-2528. [PubMed: 16825609]

46. Artsimovitch I, Svetlov V, Murakami KS, Landick R. Co-overexpression of Escherichia coli RNA polymerase subunits allows isolation and analysis of mutant enzymes lacking lineage-specific sequence insertions. J Biol Chem. 2003; 278:12344-12355. [PubMed: 12511572]

47. Vassylyeva MN, et al. The carboxy-terminal coiled-coil of the RNA polymerase beta'subunit is the main binding site for Gre factors. EMBO Rep. 2007; 8:1038-1043. [PubMed: 17917675]

48. Studitsky VM. Preparation and analysis of positioned nucleosomes. Methods Mol Biol. 1999; 119:17-26. [PubMed: 10804501] 
a

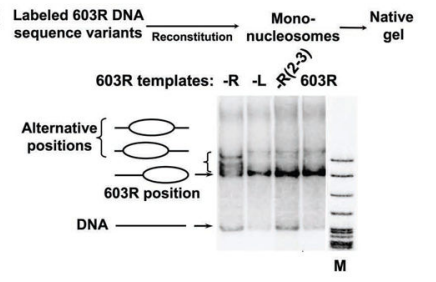

C

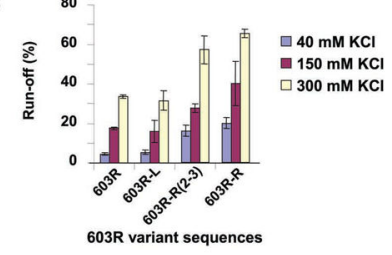

b

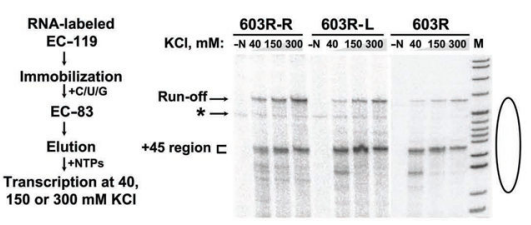

d

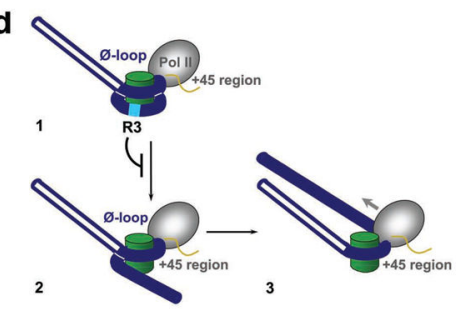

Figure 1.

Promoter-distal high-affinity (HA) nucleosome positioning sequences dictate the high nucleosomal barrier to transcription by Pol II. (a) Analysis of nucleosomes formed on the 603R variants by native PAGE. Promoter-proximal (L) or -distal (R) regions of nucleosomal DNA were mutated (Supplemental Fig. 1). The positions of the nucleosomes on the templates are indicated. M: markers, pBR322-MspI digest. (b) Mutations in the R region (603R-R template) strongly affect the nucleosomal barrier to transcription. Analysis of pulse-labeled transcripts by denaturing PAGE. The positions of labeled DNA (asterisk), transcripts and nucleosomes are indicated. (c) Mutations of 603R-R(2-3) sequence have a strong effect on the nucleosomal barrier to transcription. Run-off transcripts (Fig. 1b) were quantified and plotted. Each bar indicates the average of at least three separate experiments; error bars indicate the mean \pm S.D. (d) Pol II paused at the +45 region (1) could form a $\emptyset$ loop and sterically displace the promoter-distal DNA end from the octamer ((2) and (3)) to facilitate further transcription through a nucleosome. Strong histone-DNA contacts in the R3 HA region (Supplementary Fig. 1) would block DNA displacement and transcription. Nucleosomal DNA and the histone octamer are shown in blue and green, respectively. The direction of transcription is indicated by gray arrow. 

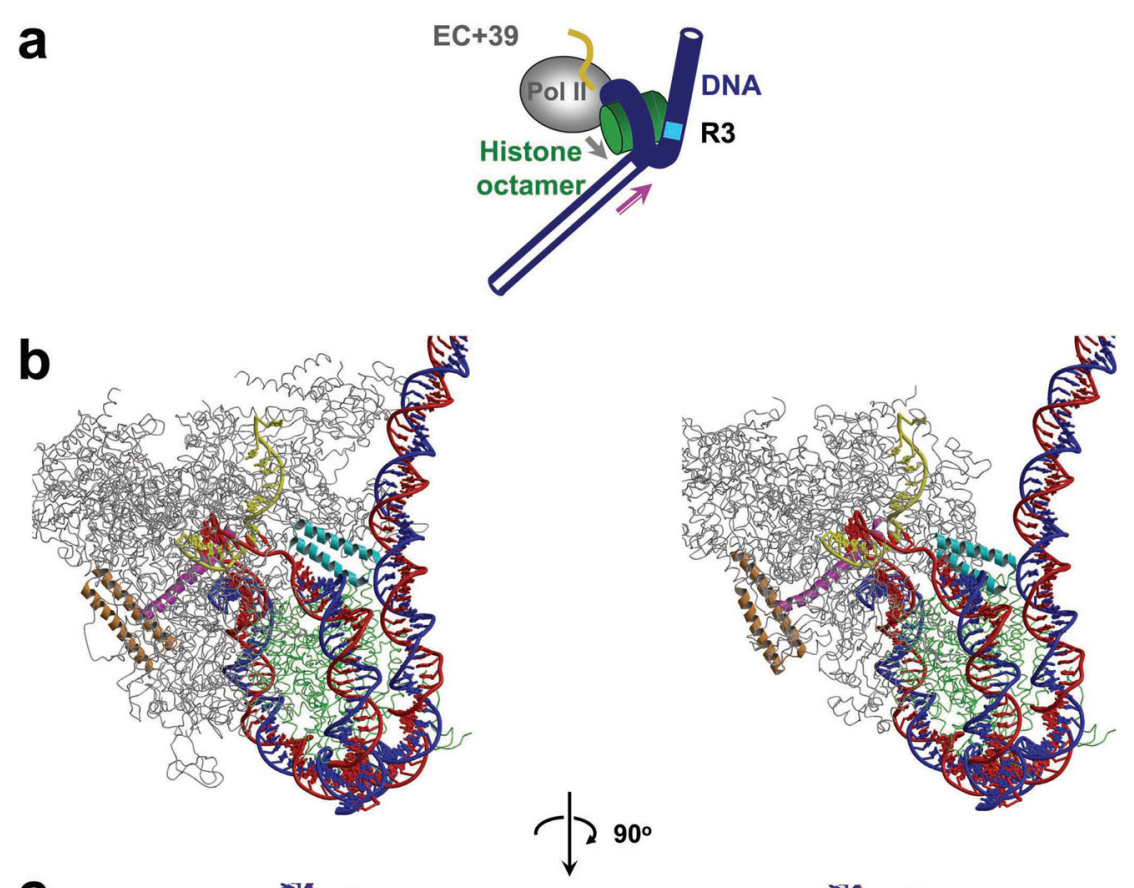

C
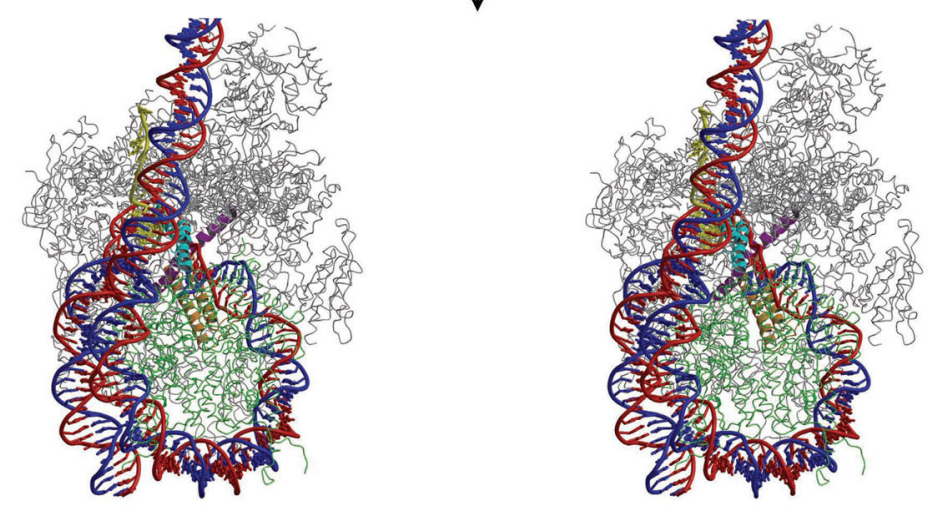

Figure 2.

A model of an intranucleosomal Pol II-containing a DNA Ø-loop. (a) Schematic representation of the structure. The DNA-histone contacts characteristic of the original nucleosome (before transcription) are established both in front of and behind the enzyme, forming a $\varnothing$-loop; the 50-bp downstream region of nucleosomal DNA including R3 HA sequence must be uncoiled from the octamer. Pink and grey arrows indicate the promoterproximal nucleosomal boundary and direction of transcription, respectively. (b) A stereo diagram of the complex. The structures of a nucleosome and yeast Pol II EC (PDB IDs 1aoi and $1 \mathrm{y} 1 \mathrm{w}$, see refs. ${ }^{28}$ and ${ }^{29}$ ) with the active site at the position +39 were merged using the docking approach. The DNA template, non-template and RNA strands are in red, blue and yellow, respectively. The Rpb2 bridge helix, the clamp, the C-terminal coiled coil and the rest of the Pol II molecule are in magenta, cyan, brown and grey, respectively. (c) The structure was rotated by $\sim 90$ degrees around the vertical axis. 

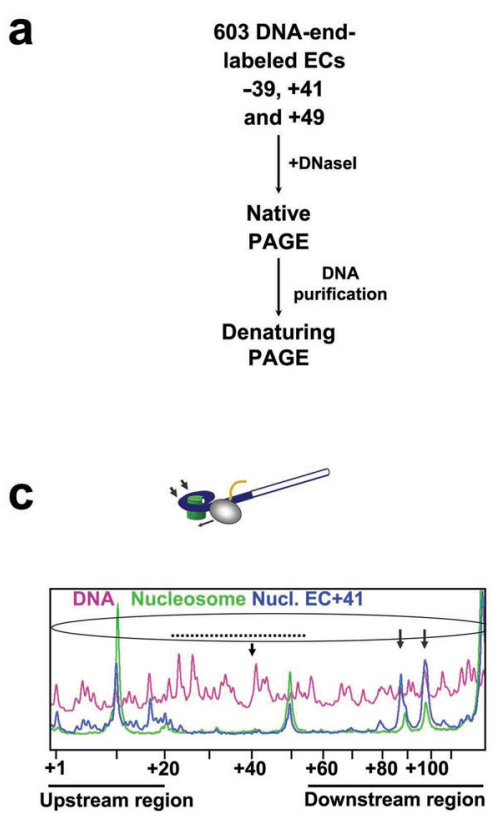
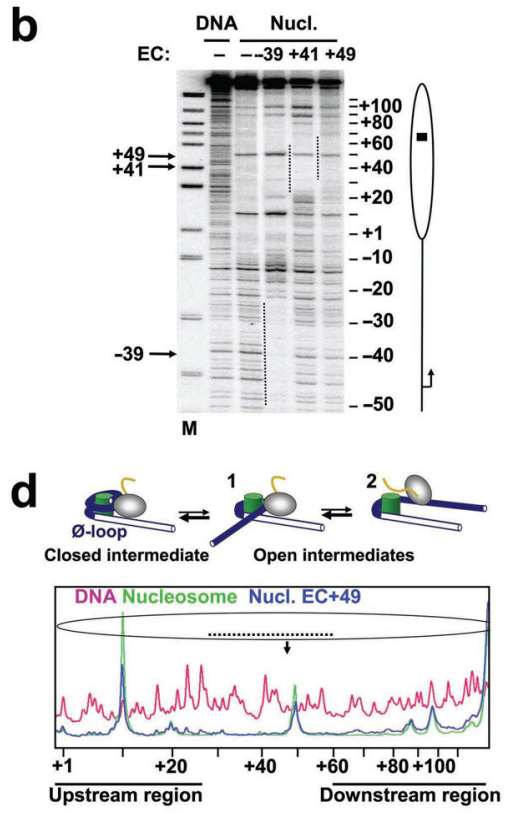
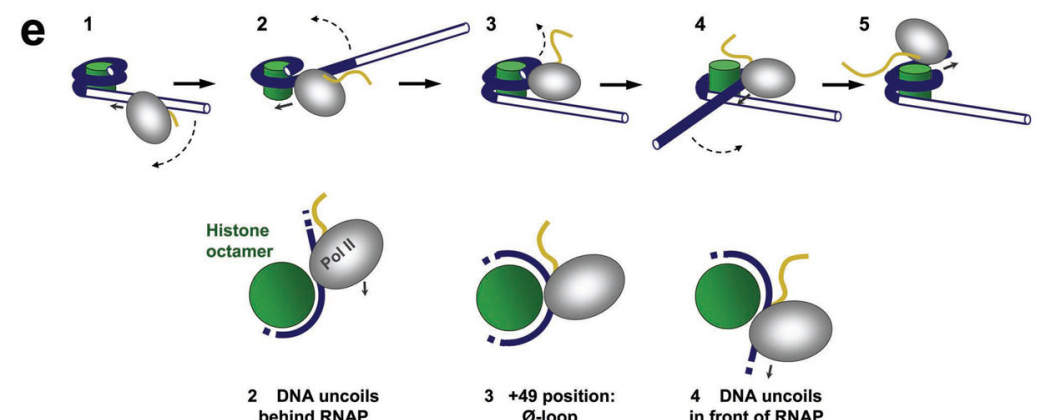

$3+49$ position:

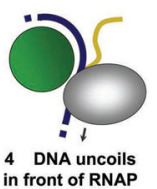

Figure 3.

RNAP forms an intranucleosomal Ø-loop at position +49 . (a) The experimental approach: DNase I footprinting of E. coli RNAP ECs. (b) Ø-loop formation is coupled with partial displacement of the promoter-distal end of the nucleosomal DNA. Analysis of end-labeled DNA by denaturing PAGE. The footprints of the ECs (dotted lines) formed on DNA and nucleosomes (Nucl.), and the positions of the active center of RNAP (arrows) are indicated. (c) and (d) Quantitative analyses of the footprints. The suggested structures of $603 \mathrm{EC}+41$ and EC+49 complexes (top). Two DNaseI-hypersensitive sites in the R3 region are indicated (arrows). (e) The Pol II-type mechanism of chromatin remodeling. As RNAP approaches a nucleosome (1), (small arrows indicate direction of transcription), upstream nucleosomal DNA is partially uncoiled (dashed arrows) from the octamer (2). Then a transient Ø-loop is formed at position +49 (3), and RNAP displaces the promoter-distal end of nucleosomal DNA (4). As RNAP continues transcription, the DNA-histone contacts upstream of the enzyme serve as an anchor to recover the nucleosome behind RNAP (5). Insert: The mechanism of nucleosome survival during transcription. Only $\sim 2 / 3$ of a DNA supercoil on the surface of the octamer is shown. 

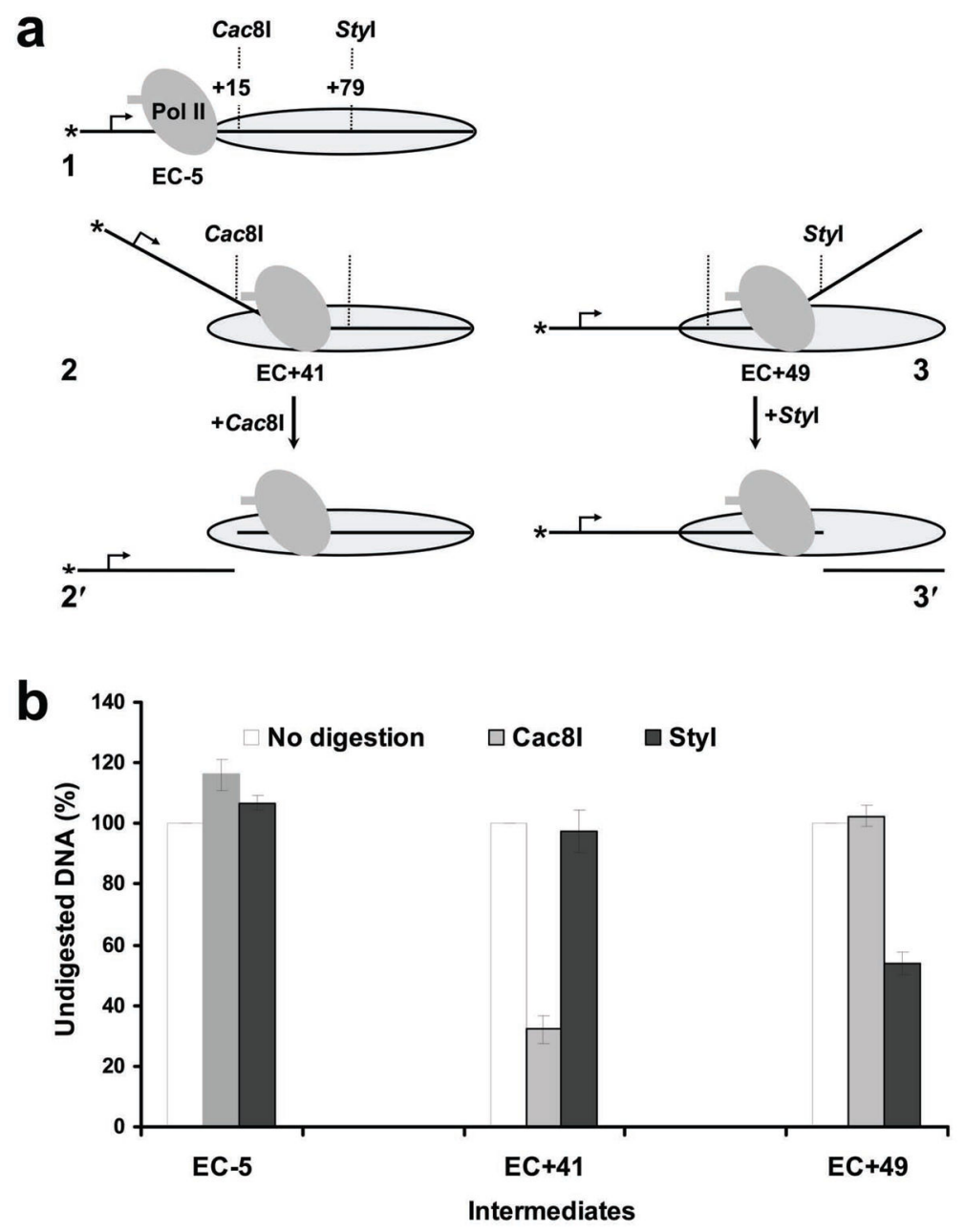

Figure 4.

Structures of the key intermediates formed during transcription through a nucleosome by Pol II. (a) Expected structures (1,2 and 3) and DNA accessibility of the complexes to Cac8I and StyI restriction enzymes $\left(2^{\prime}\right.$ and $\left.3^{\prime}\right)$. Pol II was stalled at particular positions $(-5,+42$ and +49 ) within the 603 nucleosome. The asterisk indicates the labeled DNA end. DNA is expected to be displaced from the octamer upstream of EC+41 and downstream of EC+49 (Fig. 3e). (b) Sensitivity of the ECs to restriction enzymes. ECs were incubated in the presence of an excess of $\mathrm{Cac} 8 \mathrm{I}$ or StyI restriction enzymes and analyzed on a native gel (Supplementary Fig. 5). The extents of digestion were quantified and plotted. Each bar indicates the average at least three separate experiments; error bars indicate the mean \pm S.D. 


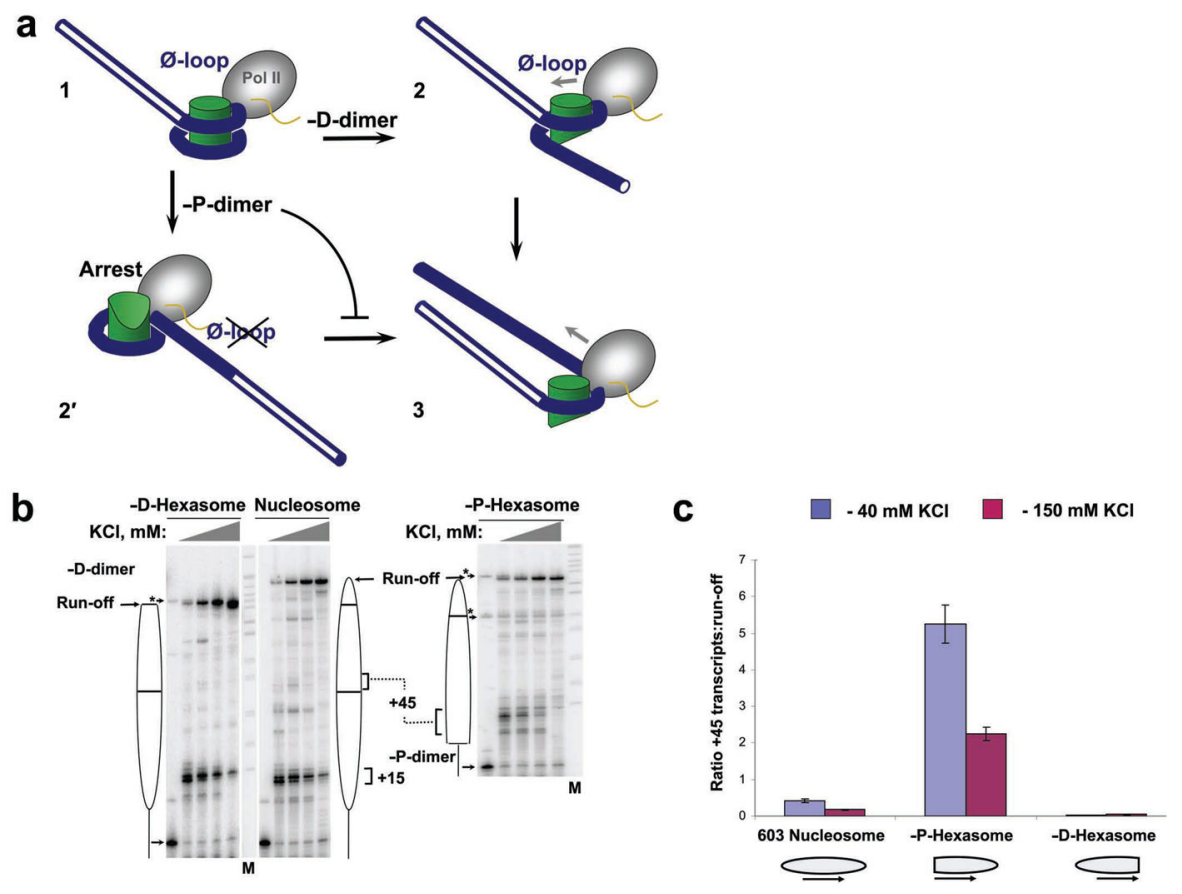

Figure 5.

Removal of the promoter-proximal $\mathrm{H} 2 \mathrm{~A} / \mathrm{H} 2 \mathrm{~B}$ dimer results in Pol II arrest in the +45 region of the nucleosome. (a) The expected consequences of removal of a single $\mathrm{H} 2 \mathrm{~A} / \mathrm{H} 2 \mathrm{~B}$ histone dimer. During Pol II progression through +45 region the $\varnothing$-loop is formed (1). Removal of the promoter-distal D-dimer promotes dissociation of the D-end of nucleosomal DNA (2) and further transcription (3), and thus would decrease the height of the +45 barrier. In contrast, removal of the proximal P-dimer favors release of the P-end of nucleosomal DNA $\left(2^{\prime}\right)$ thereby eliminating upstream DNA-histone contacts and strongly destabilizing the $\emptyset$ loop, an intermediate thought to facilitate transcription. Thus, the P-dimer removal is expected to strongly increase the height of the +45 barrier. (b) The 603 nucleosome and subnucleosomes missing either the D- or the P-dimer (-D- and -P-hexasomes, respectively) were transcribed by Pol II and transcripts were analyzed by denaturing PAGE. (c) Quantitative analysis of the efficiency of arrest in the +45 region. Each bar indicates the average at least three separate experiments; error bars indicate the mean \pm S.D. 

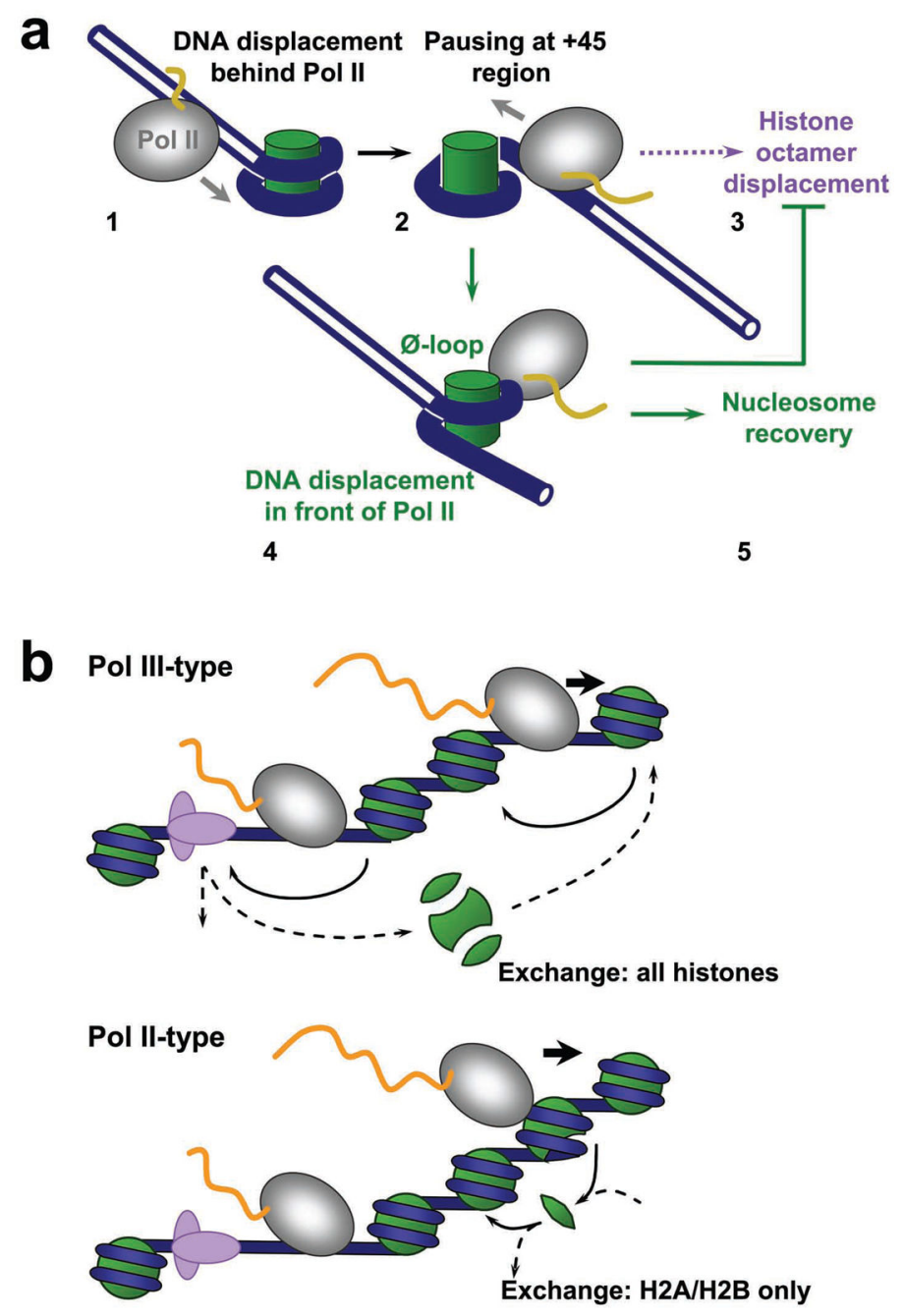

Figure 6.

A feedback mechanism allows survival of histones H3/H4 during Pol II transcription. (a) The feedback mechanism. As Pol II approaches a nucleosome (1), its progression is initially accompanied by displacement of DNA behind the enzyme (2), and could result in displacement of the histone octamer into solution (3). However when Pol II reaches the +45 region of strong DNA-histone interactions, the Ø-loop is formed (4). Ø-loop formation prevents octamer displacement and allows further transcription by facilitating DNA displacement in front of Pol II ((4) and (5)). Thus Pol II transcription is coupled with nucleosome survival. (b) Pol II-type mechanism allows survival of H3/H4 histones on DNA during transcription. Transcription by the Pol III-type mechanism would induce backward nucleosome translocation and displacement/exchange of all core histones ${ }^{23}$. In contrast, during transcription by Pol II, nucleosomes are not translocated, and only $\mathrm{H} 2 \mathrm{~A} / \mathrm{H} 2 \mathrm{~B}$ histones are exchanged. Thus, specifically modified histones $\mathrm{H} 3 / \mathrm{H} 4$ could survive Pol II transcription. 